\title{
An Exact Analysis of Heat and Mass Transfer Past a Vertical Plate with Newtonian Heating
}

\author{
Abid Hussanan, Ilyas Khan, and Sharidan Shafie \\ Department of Mathematical Sciences, Faculty of Science, Universiti Teknologi Malaysia (UTM), 81310 Skudai, Johor, Malaysia \\ Correspondence should be addressed to Sharidan Shafie; ridafie@yahoo.com
}

Received 3 February 2013; Revised 30 April 2013; Accepted 15 May 2013

Academic Editor: Mehmet Sezer

Copyright (C) 2013 Abid Hussanan et al. This is an open access article distributed under the Creative Commons Attribution License, which permits unrestricted use, distribution, and reproduction in any medium, provided the original work is properly cited.

\begin{abstract}
An exact analysis of heat and mass transfer past an oscillating vertical plate with Newtonian heating is presented. Equations are modelled and solved for velocity, temperature, and concentration using Laplace transforms. The obtained solutions satisfy governing equations and conditions. Expressions of skin friction, Nusselt number, and Sherwood number are obtained and presented in tabular forms. The results show that increasing the Newtonian heating parameter leads to increase velocity and temperature distributions whereas skin friction decreases and rate of heat transfer increases.
\end{abstract}

\section{Introduction}

Generally, the problems of free convection flows are usually modeled under the assumptions of constant surface temperature, ramped wall temperature, or constant surface heat flux [1-7]. However, in many practical situations where the heat transfer from the surface is taken to be proportional to the local surface temperature, the above assumptions fail to work. Such types of flows are termed as conjugate convective flows, and the proportionally condition of the heat transfer to the local surface temperature is termed as Newtonian heating. This work was pioneered by Merkin [8] for the free convection boundary layer flow over a vertical flat plate immersed in a viscous fluid. However, due to numerous practical applications in many important engineering devices, several other researchers are getting interested to consider the Newtonian heating condition in their problems. Few of these applications are found in heat exchanger, heat management in electrical appliances (such as computer power supplies or substation transformer), and engine cooling (such as thin fins in car radiator). Moreover, the flow over an oscillating plate with Newtonian heating also occurs in the conjugate heat transfer around fins where the conduction within the fin and the convection in the fluid surrounding it must be simultaneously analyzed in order to obtain the vital design information and in also convection flows set up when the bounding surface absorb heat by solar radiation. The literature survey shows that much attention to the problems of free convection flow with Newtonian heating is given by numerical solvers, as we can see [9-16] and the references therein. However, the exact solutions of these problems are very few [17-21]. Exact solutions on the other hand can provide an important check for numerical methods that are used to study such flows in more complex domains.

Furthermore, the free convection flows together with heat and mass transfer are of great importance in geophysics, aeronautics, and engineering. In several process such as drying, evaporation of water at body surface, energy transfer in a wet cooling tower, and flow in a desert cooler, heat and mass transfer occurs simultaneously. In view of such applications, several authors investigated free convection flows with simultaneous heat and mass transfer phenomenon [22-25].

To the best of authors' knowledge, so far, no study has been reported in the literature which investigates the unsteady free convection flow of an incompressible viscous fluid past an oscillating vertical plate with Newtonian heating and constant mass diffusion. The present study is an attempt in this direction to fill this space. In this study, the equations of the problem are first formulated and transformed into their dimensionless forms where the Laplace transform method is applied to find the exact solutions for velocity, temperature 


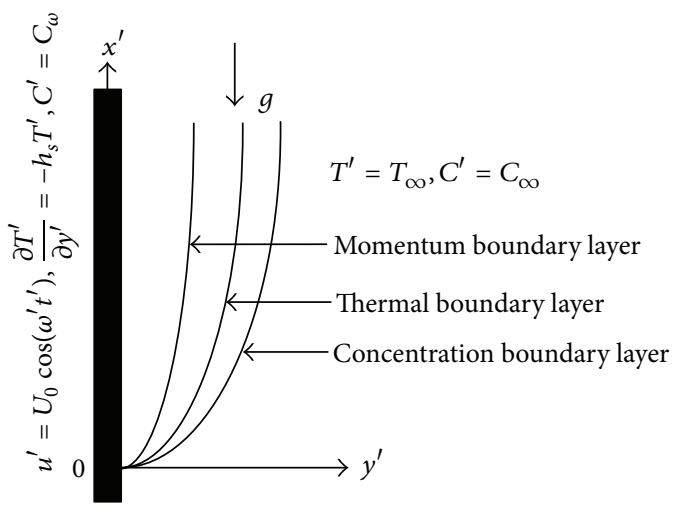

FIgure 1: Physical model and coordinate system.

and concentration. Moreover, expressions for skin friction, Nusselt number, and Sherwood number are obtained and presented in tabular forms. Finally, the obtained results are plotted graphically and discussed for the pertinent flow parameters.

\section{Mathematical Formulation}

Consider the unsteady free convection flow of a viscous incompressible fluid past a vertical plate. The $x^{\prime}$-axis is taken along the vertical plate and the $y^{\prime}$-axis is taken normal to the plate. Initially, for time $t^{\prime} \leq 0$, both the plate and fluid are at stationary condition with the constant temperature $T_{\infty}$ and concentration $C_{\infty}$. At time $t^{\prime}=0^{+}$, the plate started an oscillatory motion in its plane with the velocity $U_{0} \cos \left(\omega^{\prime} t^{\prime}\right)$ against the gravitational field, where $U_{0}$ is the amplitude of the plate oscillations. At the same time, the heat transfer from the plate to the fluid is directly proportional to the local surface temperature $T^{\prime}$ and the concentration level near the plate is raised from $C_{\infty}$ to $C_{w}$. As the plate is considered infinite in the $x^{\prime}$-axis, therefore all physical variables are independent of $x^{\prime}$ and are functions of $y^{\prime}$ and $t^{\prime}$ only. The physical model and coordinate system are presented in Figure 1.

Under the above assumptions, the governing equations of the free convective flow with Boussinesq's approximation are as follows:

$$
\begin{gathered}
\frac{\partial u^{\prime}}{\partial t^{\prime}}=\nu \frac{\partial^{2} u^{\prime}}{\partial y^{\prime 2}}+g \beta\left(T^{\prime}-T_{\infty}\right)+g \beta^{*}\left(C^{\prime}-C_{\infty}\right) \\
\rho c_{p} \frac{\partial T^{\prime}}{\partial t^{\prime}}=k \frac{\partial^{2} T^{\prime}}{\partial y^{\prime 2}}-\frac{\partial q_{r}}{\partial y^{\prime}} \\
\frac{\partial C^{\prime}}{\partial t^{\prime}}=D \frac{\partial^{2} C^{\prime}}{\partial y^{\prime 2}}
\end{gathered}
$$

The initial and boundary conditions are

$$
\begin{gathered}
t^{\prime} \leq 0: u^{\prime}=0, \quad T^{\prime}=T_{\infty}, \quad C^{\prime}=C_{\infty} \quad \forall y^{\prime} \geq 0, \\
t^{\prime}>0: u^{\prime}=U_{0} \cos \left(\omega^{\prime} t^{\prime}\right), \\
\frac{\partial T^{\prime}}{\partial y^{\prime}}=-h_{s} T^{\prime}, \quad C^{\prime}=C_{w} \quad \text { at } y^{\prime}=0, \\
u^{\prime} \longrightarrow 0, \quad T^{\prime} \longrightarrow T_{\infty}, \quad C^{\prime} \longrightarrow C_{\infty} \quad \text { as } y^{\prime} \longrightarrow \infty .
\end{gathered}
$$

The radiation heat flux under Rosseland approximation [26] is expressed by

$$
q_{r}=-\frac{4 \sigma}{3 k^{*}} \frac{\partial T^{\prime 4}}{\partial y^{\prime}}
$$

It should be noted that by using the Rosseland approximation, we limit our analysis to optically thick fluids. It is assumed that the temperature difference within the flow are sufficiently small, and then (5) can be linearized by expanding $T^{\prime 4}$ into the Taylor series about $T_{\infty}$, which after neglecting higher order terms takes the form

$$
T^{\prime 4} \cong 4 T_{\infty}^{3} T^{\prime}-3 T_{\infty}^{4} .
$$

In view of (5) and (6), (2) reduces to

$$
\rho c_{p} \frac{\partial T^{\prime}}{\partial t^{\prime}}=k\left(1+\frac{16 \sigma T_{\infty}^{3}}{3 k k^{*}}\right) T^{\prime} .
$$

To reduce the above equations into their nondimensional forms, we introduce the following nondimensional quantities:

$$
\begin{gathered}
y=\frac{y^{\prime} U_{0}}{v}, \quad t=\frac{t^{\prime} U_{0}^{2}}{v}, \quad u=\frac{u^{\prime}}{U_{0}}, \\
\theta=\frac{T^{\prime}-T_{\infty}}{T_{\infty}}, \quad C=\frac{C^{\prime}-C_{\infty}}{C_{w}-C_{\infty}}, \quad \omega=\frac{\omega^{\prime} v}{U_{0}^{2}} .
\end{gathered}
$$

Substituting (8) into (1), (7), and (3), we obtain the following nondimensional Partial Differential Equations:

$$
\begin{gathered}
\frac{\partial u}{\partial t}=\frac{\partial^{2} u}{\partial y^{2}}+\operatorname{Gr} \theta+\operatorname{Gm} C, \\
\operatorname{Pr} \frac{\partial \theta}{\partial t}=(1+R) \frac{\partial^{2} \theta}{\partial y^{2}}, \\
\operatorname{Sc} \frac{\partial C}{\partial t}=\frac{\partial^{2} C}{\partial y^{2}},
\end{gathered}
$$

where

$$
\begin{gathered}
\mathrm{Gr}=\frac{\nu g \beta T_{\infty}}{U_{0}^{3}}, \quad \mathrm{Gm}=\frac{\nu g \beta^{*}\left(C_{w}-C_{\infty}\right)}{U_{0}^{3}}, \\
\mathrm{Pr}=\frac{\mu c_{p}}{k}, \quad R=\frac{16 \sigma T_{\infty}^{3}}{3 k k^{*}}, \quad \mathrm{Sc}=\frac{v}{D},
\end{gathered}
$$

are the Grashof number, modified Grashof number, Prandtl number, radiation parameter, and Schmidt number, respectively. The corresponding initial and boundary conditions in nondimensional form are

$$
t \leq 0: u=0, \quad \theta=0, \quad C=0 \quad \forall y \geq 0,
$$

$$
t>0: u=\cos (\omega t), \frac{\partial \theta}{\partial y}=-\gamma(1+\theta), C=1 \quad \text { at } y=0
$$

$$
u \longrightarrow 0, \quad \theta \longrightarrow 0, \quad C \longrightarrow 0 \quad \text { as } y \longrightarrow \infty .
$$

Here, $\gamma=h_{s} v / U_{0}$ is the Newtonian heating parameter. We note that (12) gives $\theta=0$ when $\gamma=0$, which physically means that no heating from the plate exists $[12,16]$. 


\section{Method of Solution}

The Laplace transform method solves differential equations and corresponding initial and boundary value problems. The Laplace transform has the advantage that it solves problems directly, initial value problems without determining first a general solution and nonhomogeneous differential equations without solving first the corresponding homogeneous equations. In order to obtain the exact solution of the present problem, we will use the Laplace transform technique. Applying the Laplace transform with respect to time $t$ to the system of (9), we get

$$
\begin{aligned}
q \bar{u}(y, q)-u(y, 0)= & \frac{d^{2} \bar{u}(y, q)}{d y^{2}} \\
& +\operatorname{Gr} \bar{\theta}(y, q)+\operatorname{Gm} \bar{C}(y, q), \\
\operatorname{Pr}[q \bar{\theta}(y, q)-\theta(y, 0)]= & (1+R) \frac{d^{2} \bar{\theta}(y, q)}{d y^{2}}, \\
\operatorname{Sc}[q \bar{C}(y, q)-C(y, 0)]= & \frac{d^{2} \bar{C}(y, q)}{d y^{2}} .
\end{aligned}
$$

Here, $\bar{u}(y, q)=\int_{0}^{\infty} e^{-q t} u(y, t) d t, \bar{\theta}(y, q)=\int_{0}^{\infty} e^{-q t} \theta(y, t) d t$, and $\bar{C}(y, q)=\int_{0}^{\infty} e^{-q t} C(y, t) d t$ denote the Laplace transforms of $u(y, t), \theta(y, t)$ and $C(y, t)$, respectively.

Using the initial condition (11), we get

$$
\begin{gathered}
\frac{d^{2} \bar{u}(y, q)}{d y^{2}}-q \bar{u}(y, q)+\operatorname{Gr} \bar{\theta}(y, q)+\operatorname{Gm} \bar{C}(y, q)=0, \\
\frac{d^{2} \bar{\theta}(y, q)}{d y^{2}}-q\left(\frac{\operatorname{Pr}}{1+R}\right) \bar{\theta}(y, q)=0, \\
\frac{d^{2} \bar{C}(y, q)}{d y^{2}}-q \operatorname{Sc} \bar{C}(y, q)=0 .
\end{gathered}
$$

The corresponding transformed boundary conditions are

$$
\begin{gathered}
\bar{u}(y, q)=\frac{q}{q^{2}+\omega^{2}}, \\
\frac{d \bar{\theta}(y, q)}{d y}=-\gamma\left[\frac{1}{q}+\bar{\theta}(y, q)\right], \\
\bar{C}(y, q)=\frac{1}{q} \text { at } y=0, \\
\bar{u}(y, q) \longrightarrow 0, \quad \bar{\theta}(y, q) \longrightarrow 0, \quad \bar{C}(y, q) \longrightarrow 0
\end{gathered}
$$

The solutions of (15) subject to the boundary conditions (16) are

$$
\begin{aligned}
\bar{u}(y, q)= & \frac{1}{2(q+i \omega)} e^{-y \sqrt{q}}+\frac{1}{2(q-i \omega)} e^{-y \sqrt{q}} \\
& +\frac{a c}{q^{2}(\sqrt{q}-c)} e^{-y \sqrt{q}} \\
& -\frac{a c}{q^{2}(\sqrt{q}-c)} e^{-y \sqrt{q \mathrm{Pr}_{\mathrm{eff}}}} \\
& +\frac{b}{q^{2}} e^{-y \sqrt{q}}-\frac{b}{q^{2}} e^{-y \sqrt{q S c}} \\
\bar{\theta}(y, q)= & \frac{c}{q(\sqrt{q}-c)} e^{-y \sqrt{q \mathrm{Pr}_{\mathrm{eff}}}}, \\
\bar{C}(y, q)= & \frac{1}{q} e^{-y \sqrt{q S c}}
\end{aligned}
$$

where $a=\mathrm{Gr} /\left(\operatorname{Pr}_{\text {eff }}-1\right), b=\mathrm{Gm} /(\mathrm{Sc}-1), c=\gamma / \sqrt{\operatorname{Pr}_{\text {eff }}}$, and $\operatorname{Pr}_{\text {eff }}=\operatorname{Pr} /(1+R)$ is the effective Prandtl number defined by Magyari and Pantokratoras [27]. By taking the inverse Laplace transform of (17) and use formulae from Appendix, we obtain

$$
\begin{gathered}
\theta(y, t)=F_{4}\left(y \sqrt{\operatorname{Pr}_{\mathrm{eff}}}, t, c\right), \\
C(y, t)=F_{1}(y \sqrt{\mathrm{Sc}}, t), \\
u(y, t)=\frac{1}{2}\left[F_{5}(y, t,-i \omega)+F_{5}(y, t, i \omega)\right] \\
-\frac{a}{c^{2}}\left[F_{4}\left(y \sqrt{\operatorname{Pr}_{\mathrm{eff}}}, t, c\right)-F_{4}(y, t, c)\right] \\
+\frac{a}{c}\left[F_{2}\left(y \sqrt{\operatorname{Pr}_{\mathrm{eff}}}, t\right)-F_{2}(y, t)\right] \\
+a\left[F_{3}\left(y \sqrt{\operatorname{Pr}_{\mathrm{eff}}}, t\right)-F_{3}(y, t)\right] \\
-b\left[F_{3}(y \sqrt{\mathrm{Sc}}, t)-F_{3}(y, t)\right] .
\end{gathered}
$$

Note that the above solution is valid only for $\operatorname{Pr}_{\text {eff }} \neq 1$ and Sc $\neq 1$. Moreover, the other solutions are

Case 1. When $\mathrm{Sc}=1$ and $\operatorname{Pr}_{\text {eff }} \neq 1$,

$$
\begin{aligned}
u(y, t)= & \frac{1}{2}\left[F_{5}(y, t,-i \omega)+F_{5}(y, t, i \omega)\right] \\
& -\frac{a}{c^{2}}\left[F_{4}\left(y \sqrt{\operatorname{Pr}_{\mathrm{eff}}}, t, c\right)-F_{4}(y, t, c)\right] \\
& +\frac{a}{c}\left[F_{2}\left(y \sqrt{\mathrm{Pr}_{\mathrm{eff}}}, t\right)-F_{2}(y, t)\right] \\
& +a\left[F_{3}\left(y \sqrt{\operatorname{Pr}_{\mathrm{eff}}}, t\right)-F_{3}(y, t)\right]+\frac{y \mathrm{Gm}}{2} F_{2}(y, t) .
\end{aligned}
$$


Case 2. When $\mathrm{Sc} \neq 1$ and $\operatorname{Pr}_{\text {eff }}=1$,

$$
\begin{aligned}
u(y, t)= & \frac{1}{2}\left[F_{5}(y, t,-i \omega)+F_{5}(y, t, i \omega)\right] \\
& -b\left[F_{3}(y \sqrt{\mathrm{Sc}}, t)-F_{3}(y, t)\right]+\frac{y \mathrm{Gr}}{2 \gamma}\left[F_{4}(y, t, \gamma)\right] .
\end{aligned}
$$

Case 3. When $\mathrm{Sc}=1$ and $\operatorname{Pr}_{\text {eff }}=1$,

$$
\begin{aligned}
u(y, t)= & \frac{1}{2}\left[F_{5}(y, t,-i \omega)+F_{5}(y, t, i \omega)\right] \\
& +\frac{y \mathrm{Gr}}{2 \gamma}\left[F_{4}(y, t, \gamma)\right]-\frac{y \mathrm{Gr}}{2} \\
& \times\left[F_{2}(y, t)\right]+\frac{y \mathrm{Gm}}{2}\left[F_{2}(y, t)\right] .
\end{aligned}
$$

Here,

$$
\begin{aligned}
F_{1}(v, t)= & \operatorname{erf} c\left(\frac{v}{2 \sqrt{t}}\right), \\
F_{2}(v, t)= & 2 \sqrt{\frac{t}{\pi}} e^{-v^{2} / 4 t}-v \operatorname{erf} c\left(\frac{v}{2 \sqrt{t}}\right), \\
F_{3}(v, t)= & \left(\frac{v^{2}}{2}+t\right) \operatorname{erf} c\left(\frac{v}{2 \sqrt{t}}\right) \\
& -v \sqrt{\frac{t}{\pi}} e^{-v^{2} / 4 t}, \\
F_{4}(v, t, \alpha)= & e^{\left(\alpha^{2} t-\alpha v\right)} \operatorname{erf} c\left(\frac{v}{2 \sqrt{t}}-\alpha \sqrt{t}\right) \\
& -\operatorname{erf} c\left(\frac{v}{2 \sqrt{t}}\right), \\
F_{5}(v, t, \alpha)= & \frac{1}{2} e^{\alpha t}\left[e^{-v \sqrt{\alpha}} \operatorname{erf} c\left(\frac{v}{2 \sqrt{t}}-\sqrt{\alpha t}\right)\right. \\
\quad+e^{v \sqrt{\alpha}} & \left.\operatorname{erf} c\left(\frac{v}{2 \sqrt{t}}+\sqrt{\alpha t}\right)\right],
\end{aligned}
$$

where erf $c(\cdot)$ is the complementary error function, $v$ and $\alpha$ are dummy variables, and $F_{1}, F_{2}, F_{3}, F_{4}$, and $F_{5}$ are dummy functions.

The dimensionless expression for skin friction evaluated from (20) is given by

$$
\begin{aligned}
\tau= & \frac{\tau^{\prime}}{\rho U_{0}^{2}}=-\left.\frac{\partial u}{\partial y}\right|_{y=0} \\
= & \frac{1}{2}\left[e^{-i \omega t} \sqrt{-i \omega} \operatorname{erf}(\sqrt{-i \omega t})\right. \\
& \left.+e^{i \omega t} \sqrt{i \omega} \operatorname{erf}(\sqrt{i \omega t})\right] \\
& +\frac{a}{c}\left[1-e^{c^{2} t}(1+\operatorname{erf}(c \sqrt{t}))\right] \\
& \times\left(\sqrt{\operatorname{Pr}_{\mathrm{eff}}}-1\right)+\frac{1}{\sqrt{\pi t}} \\
& +2 \sqrt{\frac{\operatorname{Pr}_{\mathrm{eff}} t}{\pi}}-2 a \sqrt{\frac{t}{\pi}}-2 b \sqrt{\frac{t}{\pi}}(\sqrt{\mathrm{Sc}}-1) .
\end{aligned}
$$

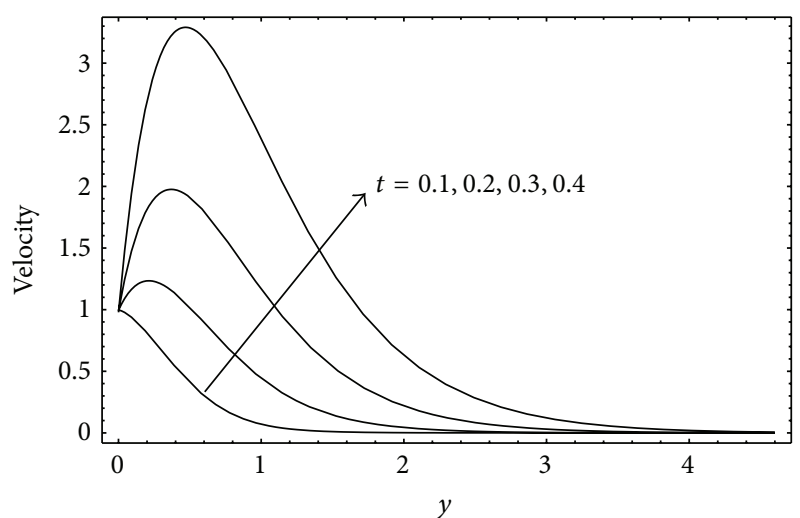

FIGURE 2: Velocity profiles for different values of $t$ when $R=2, \operatorname{Pr}=$ $0.71, \mathrm{Gr}=5, \mathrm{Gm}=1, \mathrm{Sc}=0.78, \gamma=1$, and $\omega=\pi / 6$.

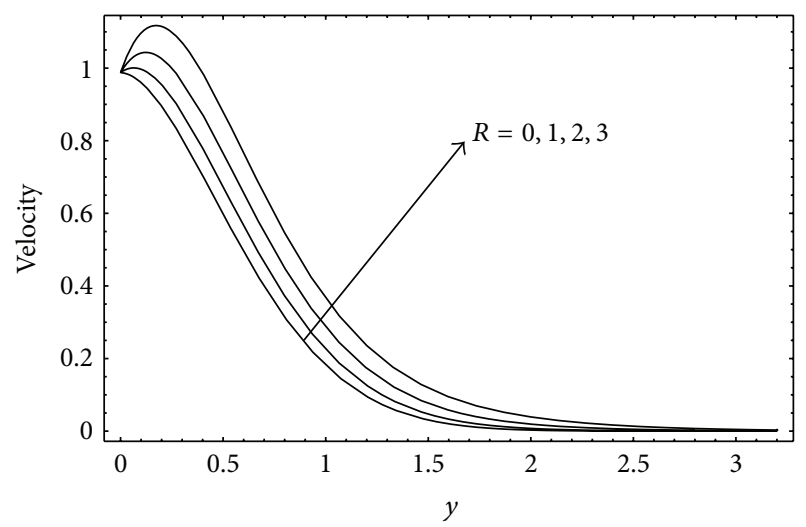

FIGURE 3: Velocity profiles for different values of $R$ when $t=$ $0.2, \mathrm{Pr}=0.71, \mathrm{Gr}=2, \mathrm{Gm}=3, \mathrm{Sc}=0.94, \gamma=1$, and $\omega=\pi / 4$. by

The dimensionless expression of Nusselt number is given

$$
\begin{aligned}
\mathrm{Nu} & =-\left.\frac{v}{U_{0}\left(T^{\prime}-T_{\infty}\right)} \frac{\partial T^{\prime}}{\partial y^{\prime}}\right|_{y^{\prime}=0}=\frac{1}{\theta(0, t)}+1 \\
& =c \sqrt{\operatorname{Pr}_{\text {eff }}}\left(1+\frac{1}{e^{c^{2} t}[1+\operatorname{erf}(c \sqrt{t})]-1}\right) .
\end{aligned}
$$

The dimensionless expression of Sherwood number is given by

$$
\mathrm{Sh}=-\left.\frac{\partial C}{\partial y}\right|_{y=0}=\sqrt{\frac{\mathrm{Sc}}{\pi t}}
$$

\section{Graphical Results and Discussion}

In order to reveal some relevant physical aspects of the obtained solutions, the numerical results for velocity, temperature, and concentration are computed and shown graphically in Figures 2-16, to illustrate the influence of embedded flow parameters such as time $t$, radiation parameter $R$, Prandtl number Pr, Grashof number Gr, modified Grashof 
TABLE 1: Skin friction variation.

\begin{tabular}{lcccccccc}
\hline$t$ & $R$ & Pr & Gr & Gm & Sc & $\gamma$ & $\omega$ & $\tau$ \\
\hline 0.01 & 1 & 0.71 & 5 & 2 & 0.22 & 1 & $\pi / 2$ & 5.4263 \\
0.02 & 1 & 0.71 & 5 & 2 & 0.22 & 1 & $\pi / 2$ & 3.6395 \\
0.01 & 2 & 0.71 & 5 & 2 & 0.22 & 1 & $\pi / 2$ & 5.4049 \\
0.01 & 1 & 1.0 & 5 & 2 & 0.22 & 1 & $\pi / 2$ & 5.4401 \\
0.01 & 1 & 0.71 & 10 & 2 & 0.22 & 1 & $\pi / 2$ & 5.3663 \\
0.01 & 1 & 0.71 & 5 & 4 & 0.22 & 1 & $\pi / 2$ & 5.2727 \\
0.01 & 1 & 0.71 & 5 & 2 & 0.62 & 1 & $\pi / 2$ & 5.4537 \\
0.01 & 1 & 0.71 & 5 & 2 & 0.22 & 2 & $\pi / 2$ & 5.3473 \\
0.01 & 1 & 0.71 & 5 & 2 & 0.22 & 1 & $\pi$ & 5.4208 \\
\hline
\end{tabular}

TABLE 2: Nusselt number variation.

\begin{tabular}{lcccc}
\hline$t$ & $R$ & $\mathrm{Pr}$ & $\gamma$ & $\mathrm{Nu}$ \\
\hline 0.2 & 2 & 0.71 & 1 & 1.3119 \\
0.4 & 2 & 0.71 & 1 & 1.1054 \\
0.2 & 4 & 0.71 & 1 & 1.1471 \\
0.2 & 2 & 1.0 & 1 & 1.4659 \\
0.2 & 2 & 0.71 & 2 & 2.0347 \\
\hline
\end{tabular}

TABLE 3: Sherwood number variation.

\begin{tabular}{lcc}
\hline$t$ & Sc & Sh \\
\hline 0.2 & 0.22 & 0.5917 \\
0.4 & 0.22 & 0.4184 \\
0.2 & 0.62 & 0.9938 \\
\hline
\end{tabular}

number Gm, Schmidt number Sc, Newtonian heating parameter $\gamma$, and phase angle $\omega t$. The numerical values for skin friction, Nusselt number, and Sherwood number for these parameters are also presented in Tables 1-3.

The velocity profiles for different values of time $t$ are shown in Figure 2. It is observed that the velocity increases with increasing values of time $t$. The velocity profiles in case of radiation and pure convection are shown in Figure 3. It is found from this figure that the radiation parameter $R$ has an accelerating effect on velocity. Physically, it is due to the fact that an increase in the radiation parameter $R$ for fixed values of other parameters decreases the rate of radiative heat transfer to the fluid, and consequently, the fluid velocity increases. This behavior of $R$ is quite identical with that found in Figure 6 of Mohamed et al. [28].

The effects of Prandtl number Pr on the velocity profiles are shown in Figure 4 for $\operatorname{Pr}=0.71$ (air), $\operatorname{Pr}=1.0$ (electrolytic solution), $\operatorname{Pr}=7.0$ (water), and $\operatorname{Pr}=100$ (engine oil). It is seen from this figure that an increase in the values of Prandtl number Pr results in the decrease of velocity. In heat transfer analysis, the role of Prandtl number Pr is to control the relative thickness of the momentum and thermal boundary layers. For small value of Pr the heat diffuses very quickly compared to the velocity. This means that for liquid metals, the thickness of the thermal boundary layer is much bigger than the velocity boundary layer. The effects of Grashof number $\mathrm{Gr}$ and modified Grashof number $\mathrm{Gm}$ on velocity are shown in Figures 5 and 6. It is found that the effects of Grashof

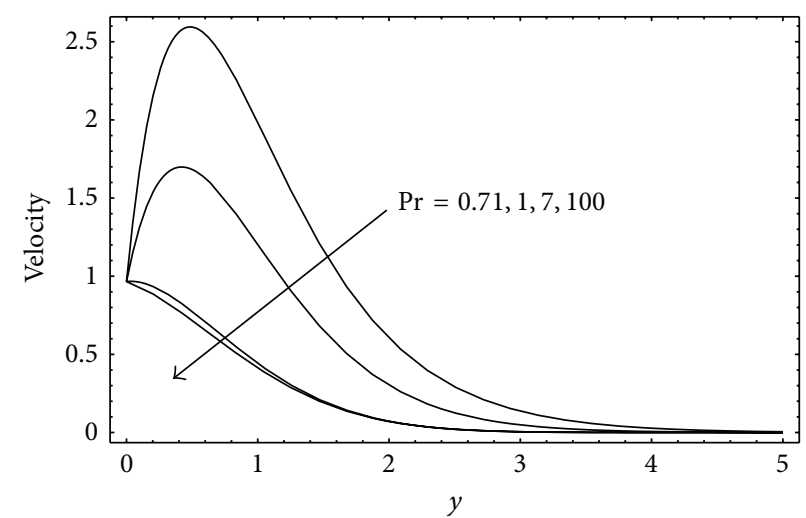

FIGURE 4: Velocity profiles for different values of Pr when $t=$ $0.5, R=2, \mathrm{Gr}=2, \mathrm{Gm}=1, \mathrm{Sc}=0.78, \gamma=1$, and $\omega=\pi / 6$.

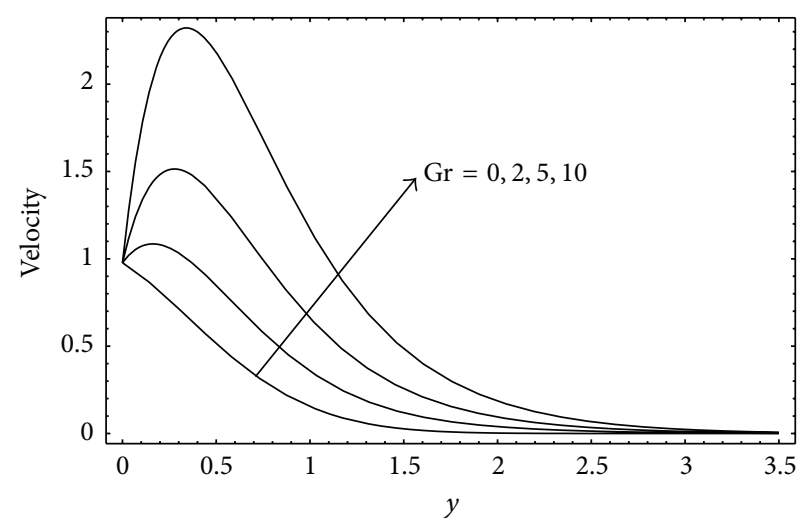

FIGURE 5: Velocity profiles for different values of $\mathrm{Gr}$ when $t=$ $0.2, R=3, \operatorname{Pr}=0.71, \mathrm{Gm}=2, \mathrm{Sc}=0.78, \gamma=1$, and $\omega=\pi / 3$.

number Gr and modified Grashof number Gm on velocity are similar. Velocity increases with increasing values of $\mathrm{Gr}$ and $\mathrm{Gm}$. Physically, it is possible because an increase in the values of Grashof number Gr and modified Grashof number Gm has the tendency to increase the thermal and mass buoyancy effects. This gives rise to an increase in the induced flow. Further, from these figures, it is noticed that Grashof number and modified Grashof number do not have any influence as the fluid move away from the bounding surface.

The effects of Sc on the velocity profiles are shown in Figure 7. Four different values of Schmidt number Sc = $0.22,0.62,0.78$, and 0.94 are chosen. They physically correspond to hydrogen, water vapour, ammonia, and carbon dioxide, respectively. It is clear that the velocity decreases as the Schmidt number Sc increases. Further, it is clear from this figure that the velocity for hydrogen is the maximum and carbon dioxide carries the minimum velocity. Figure 8 displays the effect of Newtonian heating parameter $\gamma$ on the dimensionless velocity. It is found that as the Newtonian heating parameter increases, the density of the fluid decreases, and the momentum boundary layer thickness increases and as a result, and the velocity increases within the boundary layer.

The graphical results for the phase angle $\omega t$ are shown in Figure 9. It is interesting to note that when the phase 


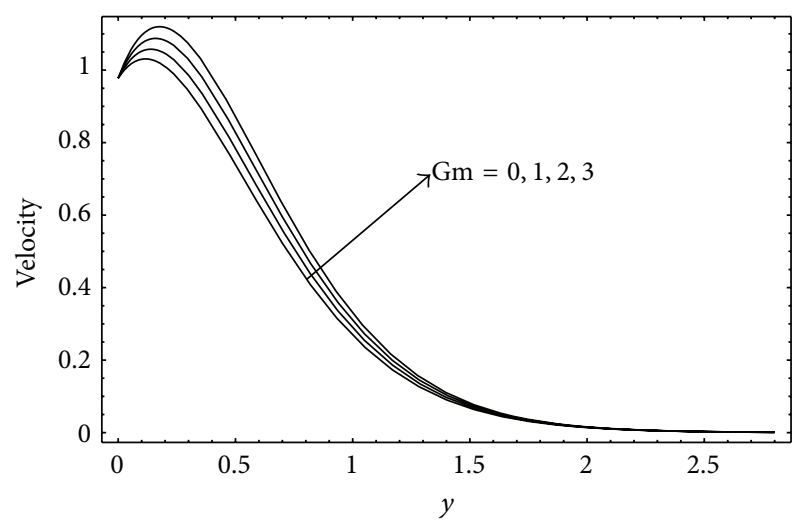

Figure 6: Velocity profiles for different values of $\mathrm{Gm}$ when $t=$ $0.2, R=1, \operatorname{Pr}=0.71, \mathrm{Gr}=5, \mathrm{Sc}=0.78, \gamma=1$, and $\omega=\pi / 3$.

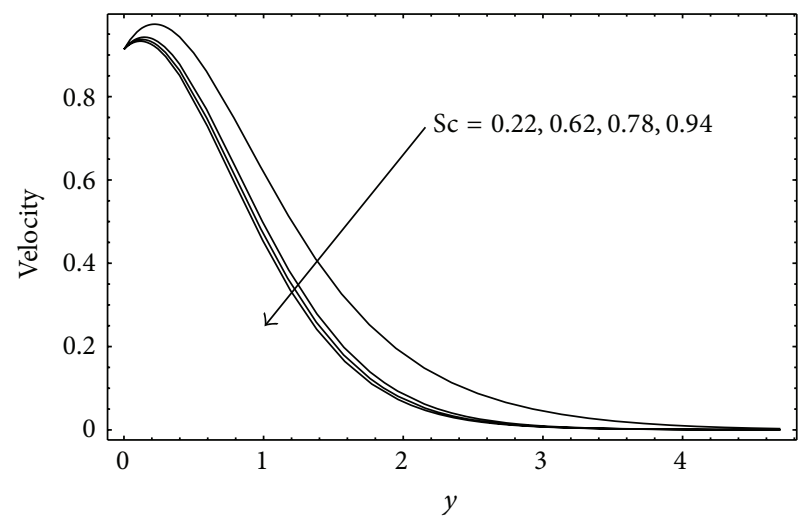

FIgURE 7: Velocity profiles for different values of Sc when $t=$ $0.4, R=2, \operatorname{Pr}=0.71, \mathrm{Gr}=5, \mathrm{Gm}=2, \gamma=0.1$, and $\omega=\pi / 3$.

angle $\omega t$ is zero which physically corresponds to no oscillation, then the fluid approaches to its maximum velocity of magnitude 1 meter per second, whereas for the phase angle $\omega t=\pi / 2$, the velocity gains its minimum value of magnitude 0 meter per second. The oscillations near the plate are of great significance; however, these oscillations reduce for large values of the independent variable $y$ and approach to zero as $y$ tends to infinity. The velocity profiles are plotted in Figure 10 for different values of Sc when $\omega=0$ (impulsive motion of the plate). It is found from this figure that the behavior of Sc on the velocity profiles quite identical with that found in Figure 6 of Narahari and Nayan [20]. Further, all these graphical results discussed above are in good agreement with the imposed boundary conditions given by (12) and (13). Hence, this ensures the accuracy of our results.

The effects of various parameters on the temperature and concentration profiles are shown in Figures 11-16. In these figures, Figure 11 exhibits the influence of dimensionless time $t$ on the temperature. It is found that the temperature profiles increase with increasing time. From Figure 12, it is noted that an increase in the radiation parameter $R$ leads to an increase in the temperature due to the fact that thermal boundary layer thickness of fluid increases. The influence of Prandtl number Pr on temperature profiles is shown in

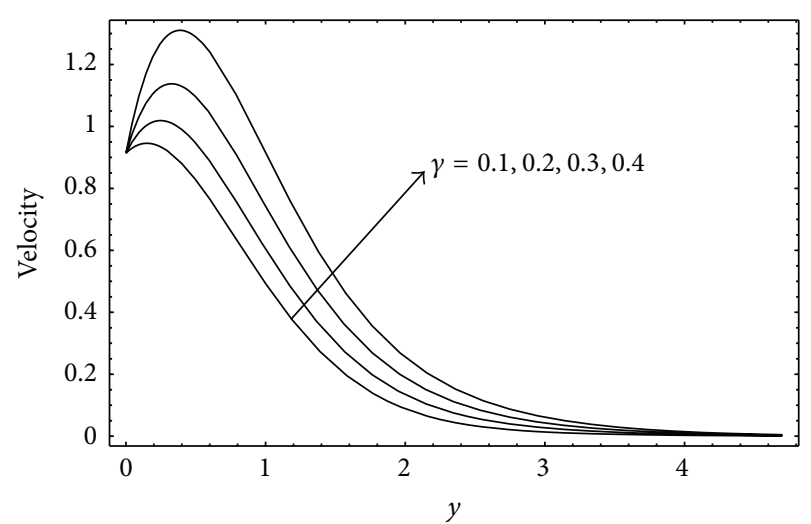

FIGURE 8: Velocity profiles for different values of $\gamma$ when $t=$ $0.4, R=3, \mathrm{Pr}=0.71, \mathrm{Gr}=5, \mathrm{Gm}=2, \mathrm{Sc}=0.78$, and $\omega=\pi / 3$.

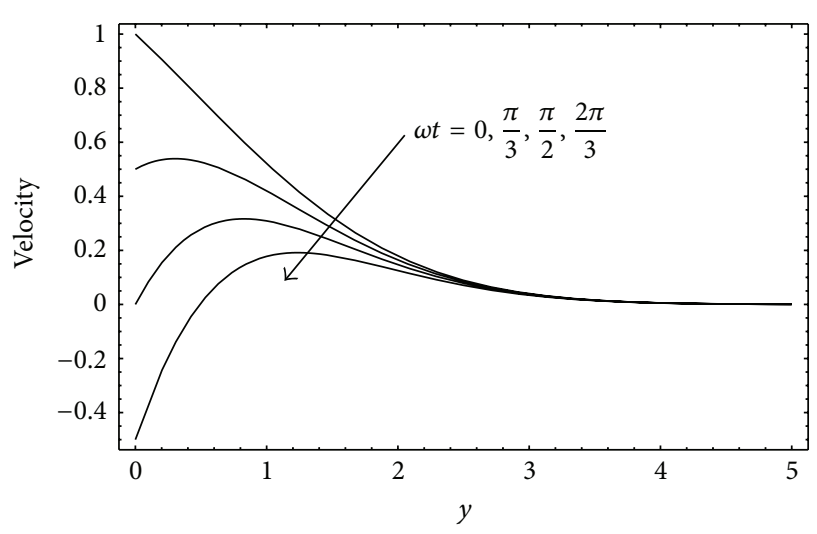

Figure 9: Velocity profiles for different values of $\omega t$ when $t=1, R=$ $0.5, \mathrm{Pr}=100, \mathrm{Gr}=2, \mathrm{Gm}=0.2, \mathrm{Sc}=0.94$, and $\gamma=0.01$.

Figure 13. It is found that the temperature decreases as the Prandtl number Pr increases. Physically, the increase of Pr means the decrease of thermal conductivity of fluid. From Figure 14, it is observed that an increase in the Newtonian heating parameter increases the thermal boundary layer thickness and as a result the surface temperature of the plate increases. On the other hand, it is found from Figure 15 that the influence of time $t$ on concentration profiles is similar to the velocity and temperature profiles given in Figures 2 and 11. The effects of Schmidt number Sc on the concentration profiles are shown in Figure 16. It is seen from this figure that an increase in the value of Schmidt number makes the concentration boundary layer thin, and hence, the concentration profiles decrease.

The numerical results for skin friction, Nusselt number, and Sherwood number are shown in Tables 1, 2, and 3 for various parameters of interest. It is depicted from Table 1 that skin friction decreases with, increasing $t, R, \mathrm{Gr}, \mathrm{Gm}, \gamma$ and $\omega t$, while it increases as $\operatorname{Pr}$ and Sc are increased. Table 2 reveals that the Nusselt number increases as $\operatorname{Pr}$ and $\gamma$ are increased and decreases when $t$ and $R$ are increased. From Table 3, it is observed that the Sherwood number increases with increasing Sc, while reverse effect is observed for $t$. 
TABLE 4: Inverse Laplace transform formulae.

\begin{tabular}{ccc}
\hline & $F(q)=L\{f(t)\}$ & $f(t)$ \\
1 & $\frac{1}{q} e^{-y \sqrt{q}}$ & $\operatorname{erf} c\left(\frac{y}{2 \sqrt{t}}\right)$ \\
2 & $\frac{1}{q^{2}} e^{-y \sqrt{q}}$ & $\left(\frac{y^{2}}{2}+t\right) \operatorname{erf} c\left(\frac{y}{2 \sqrt{t}}\right)-y \sqrt{\frac{t}{\pi}} e^{-y^{2} / 4 t}$ \\
3 & $\frac{1}{q \sqrt{q-a}} e^{-y \sqrt{q}}$ & $e^{\left(a^{2} t-a y\right)} \operatorname{erf} c\left(\frac{y}{2 \sqrt{t}}-a \sqrt{t}\right)-\operatorname{erf} c\left(\frac{y}{2 \sqrt{t}}\right)$ \\
4 & $\frac{1}{q^{2} \sqrt{q-a}} e^{-y \sqrt{q}}$ & $\frac{1}{4 \sqrt{\pi t}} e^{-y^{2} / 4 t}+a e^{\left(a^{2} t-a y\right)} \operatorname{erf} c\left(\frac{y}{2 \sqrt{t}}-a \sqrt{t}\right)$ \\
5 & $\frac{1}{q+a} e^{-y \sqrt{q}}$ & $\frac{e^{a t}}{2} e^{y \sqrt{a}} \operatorname{erf} c\left(\frac{y}{2 \sqrt{t}}+\sqrt{a t}\right)+e^{-y \sqrt{a}} \operatorname{erf} c\left(\frac{y}{2 \sqrt{t}}-\sqrt{a t}\right)$
\end{tabular}

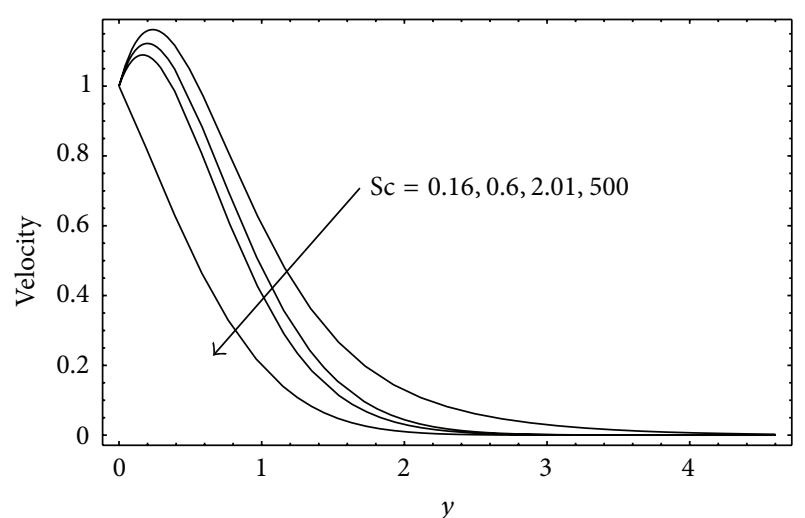

Figure 10: Velocity profiles for different values of Sc when $t=$ $0.3, R=0.5, \operatorname{Pr}=0.71, \mathrm{Gr}=3, \mathrm{Gm}=2, \gamma=1$, and $\omega=0$.

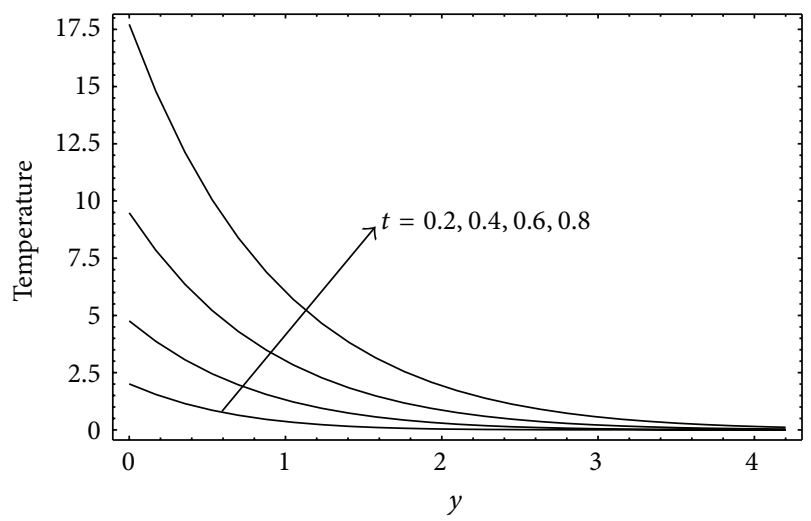

Figure 11: Temperature profiles for different values of $t$ when $R=$ $1, \operatorname{Pr}=0.71$, and $\gamma=1$.

\section{Conclusions}

In this paper, exact solutions of unsteady free convection flow of an incompressible viscous fluid past an oscillating vertical plate with Newtonian heating and constant mass diffusion are obtained using Laplace transform technique. The results obtained show that the velocity and temperature are increased with increasing Newtonian heating parameter. Further, the effect of Newtonian heating parameter increases the Nusselt number but reduces the skin friction. However, the Nusselt number is decreased when the radiation parameter is increased. Also, the skin friction is decreased when

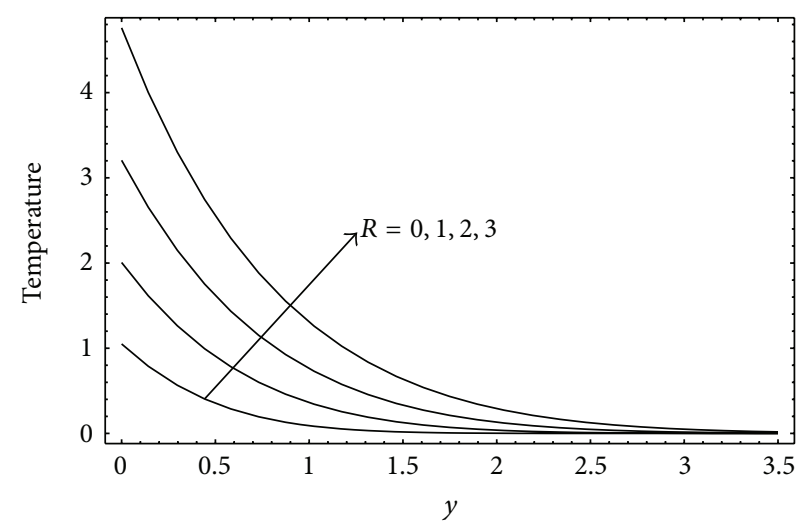

FIGURE 12: Temperature profiles for different values of $R$ when $t=$ $0.2, \operatorname{Pr}=0.71$, and $\gamma=1$.

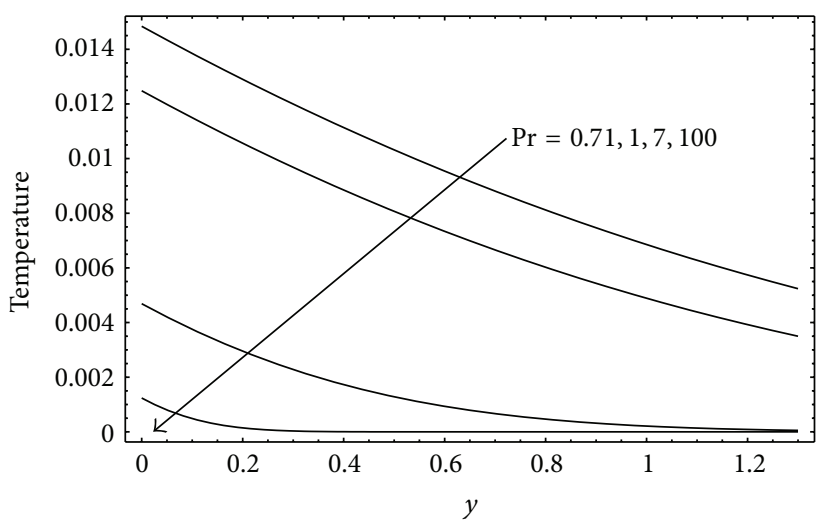

FIGURE 13: Temperature profiles for different values of $\operatorname{Pr}$ when $t=$ $0.2, R=5$, and $\gamma=0.01$.

the radiation parameter, phase angle and Grashof number are increased. The exact solutions obtained in this study are significant not only because they are solutions of some fundamental flows, but also they serve as accuracy standards for approximate methods, whether numerical, asymptotic, or experimental.

\section{Appendix}

See Table 4. 


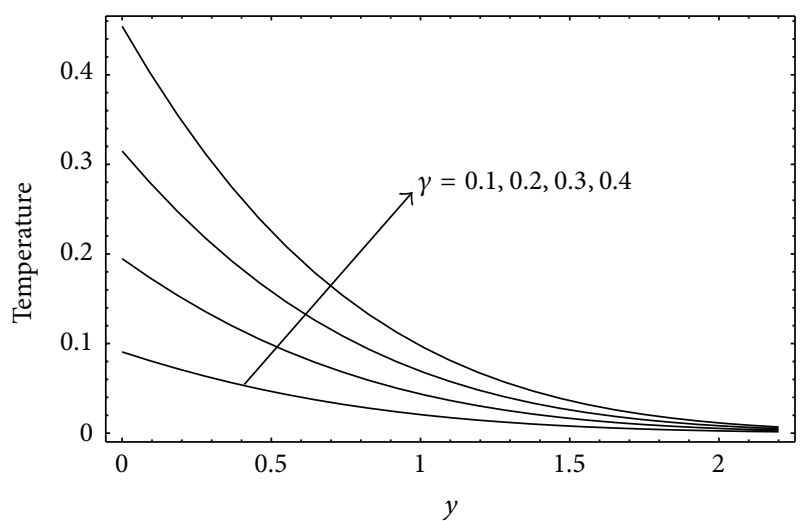

Figure 14: Temperature profiles for different values of $\gamma$ when $t=$ $0.2, R=1$, and $\operatorname{Pr}=0.71$.

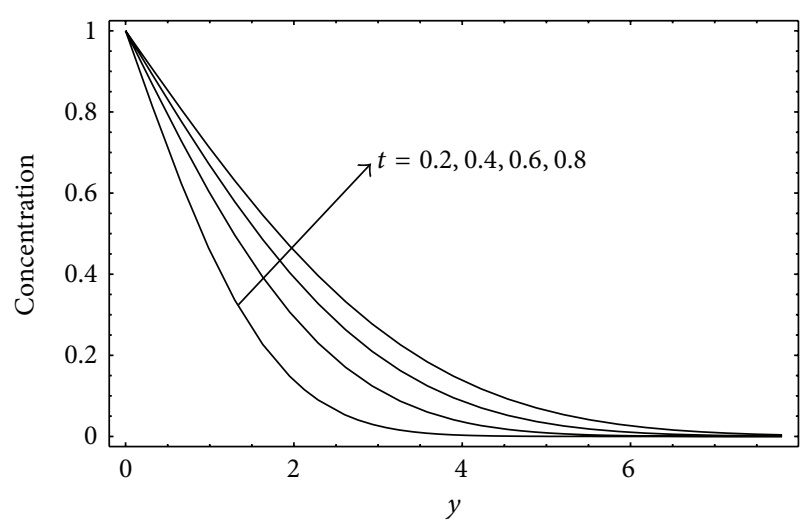

FIGURE 15: Concentration profiles for different values of $t$ when $\mathrm{Sc}=$ 0.22 .

\section{Nomenclature}

$C^{\prime}$ : Species concentration in the fluid

$C_{w}$ : Species concentration near the plate

$C_{\infty}$ : Species concentration in the fluid far away from the plate

C: Dimensionless concentration

$c_{p}$ : Heat capacity at a constant pressure

D: Mass diffusivity

g: Acceleration due to gravity

$h_{s}$ : Heat transfer parameter for Newtonian heating

Gr: Thermal Grashof number

Gm: Modified Grashof number

$k$ : Thermal conductivity of the fluid

Pr: Prandtl number

$q_{r}$ : Radiative heat flux in the $y^{\prime}$-direction

$R: \quad$ Radiation parameter

Sc: Schmidt number

$T^{\prime}$ : Temperature of the fluid

$T_{\infty}$ : Ambient temperature

$t^{\prime}:$ Time

$t$ : Dimensionless time

$u^{\prime}$ : Velocity of the fluid in the $x^{\prime}$-direction

$u$ : Dimensionless velocity

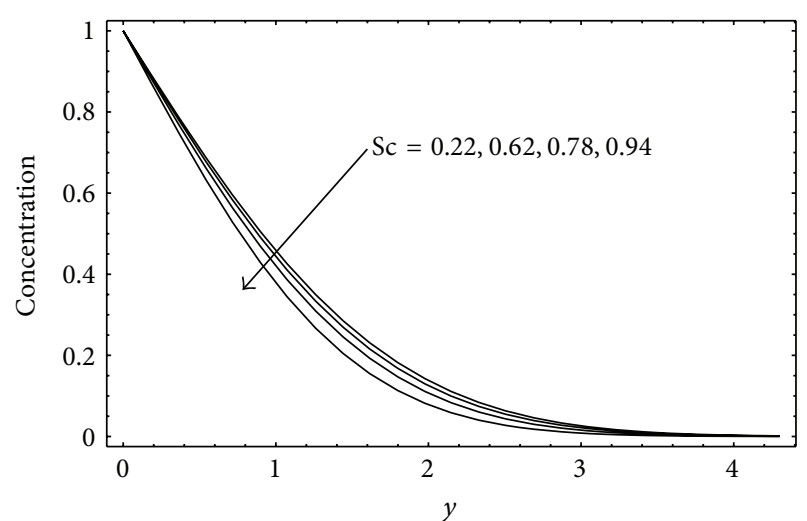

Figure 16: Concentration profiles for different values of Sc when $t=0.2$.

$y^{\prime}: \quad$ Coordinate axis normal to the plate

$y: \quad$ Dimensionless coordinate axis normal to the plate

$k$ : Thermal conductivity

$k^{*}$ : Mean absorption coefficient

$\beta$ : $\quad$ Volumetric coefficient of thermal expansion

$\beta^{*}$ : Volumetric coefficient of mass expansion

$\mu$ : $\quad$ Coefficient of viscosity

$\nu$ : Kinematic viscosity

$\rho: \quad$ Fluid density

$\sigma: \quad$ Stefan-Boltzmann constant

$\tau: \quad$ Skin friction

$\tau^{\prime}: \quad$ Dimensionless skin friction

$\theta: \quad$ Dimensionless temperature

$\omega^{\prime}: \quad$ Frequency of oscillation

$\omega t$ : Phase angle

erf $c$ : Complementary error function.

\section{Acknowledgments}

The authors would like to acknowledge MOHE and Research Management Centre, UTM for the financial support through Vote nos. 4F109 and 04H27 for this research.

\section{References}

[1] P. Chandrakala, "Radiation effects on flow past an impulsively started vertical oscillating plate with uniform heat flux," International Journal of Dynamics of Fluids, vol. 6, pp. 209-215, 2010.

[2] R. K. Deka and S. K. Das, "Radiation effects on free convection flow near a vertical plate with ramped wall temperature," Engineering, vol. 3, pp. 1197-1206, 2011.

[3] P. Chandran, N. C. Sacheti, and A. K. Singh, "Natural convection near a vertical plate with ramped wall temperature," Heat and Mass Transfer, vol. 41, no. 5, pp. 459-464, 2005.

[4] V. M. Soundalgekar, R. M. Lahurikar, S. G. Pohanerkar, and N. S. Birajdar, "Effects of mass transfer on the flow past an oscillating infinite vertical plate with constant heat flux," Thermophysics and AeroMechanics, vol. 1, pp. 119-124, 1994. 
[5] V. M. Soundalgekar, R. M. Lahurikar, S. G. Pohanerkar, and N. S. Birajdar, "Mass transfer effects on flow past a vertical oscillating plate with variable temperature," Heat and Mass Transfer, vol. 30, no. 5, pp. 309-312, 1995.

[6] G. Pathak, C. H. Maheshwari, and S. P. Gupta, "Effects of radiation on unsteady free convection flow bounded by an oscillating plate with variable temperature," International Journal of Applied Mechanics and Engineering, vol. 11, pp. 371-382, 2006.

[7] R. Muthucumaraswamy, "Natural convection on flow past an impulsively started vertical plate with variable surface heat flux," Far East Journal of Applied Mathematics, vol. 14, no. 1, pp. 99119, 2004.

[8] J. H. Merkin, "Natural-convection boundary-layer flow on a vertical surface with Newtonian heating," International Journal of Heat and Fluid Flow, vol. 15, no. 5, pp. 392-398, 1994.

[9] D. Lesnic, D. B. Ingham, and I. Pop, "Free convection boundarylayer flow along a vertical surface in a porous medium with Newtonian heating," International Journal of Heat and Mass Transfer, vol. 42, no. 14, pp. 2621-2627, 1999.

[10] D. Lesnic, D. B. Ingham, and I. Pop, "Free convection from a horizontal surface in a porous medium with newtonian heating," Journal of Porous Media, vol. 3, no. 3, pp. 227-235, 2000.

[11] D. Lesnic, D. B. Ingham, I. Pop, and C. Storr, "Free convection boundary-layer flow above a nearly horizontal surface in a porous medium with newtonian heating," Heat and Mass Transfer, vol. 40, no. 9, pp. 665-672, 2004.

[12] M. Z. Salleh, R. Nazar, and I. Pop, "Boundary layer flow and heat transfer over a stretching sheet with Newtonian heating," Journal of the Taiwan Institute of Chemical Engineers, vol. 41, no. 6, pp. 651-655, 2010.

[13] M. Z. Salleh, R. Nazar, and I. Pop, "Numerical solutions of free convection boundary layer flow on a solid sphere with Newtonian heating in a micropolar fluid," Meccanica, vol. 47, pp. 1261-1269, 2012.

[14] M. Z. Salleh, R. Nazar, N. M. Arifin, I. Pop, and J. H. Merkin, "Forced-convection heat transfer over a circular cylinder with Newtonian heating," Journal of Engineering Mathematics, vol. 69, no. 1, pp. 101-110, 2011.

[15] S. Das, C. Mandal, and R. N. Jana, "Radiation effects on unsteady free convection flow past a vertical plate with Newtonian heating," International Journal of Computer Applications, vol. 41, pp. 36-41, 2012.

[16] A. R. M. Kasim, N. F. Mohammad, Aurangzaib, and S. Sharidan, "Natural convection boundary layer flow of a viscoelastic fluid on solid sphere with Newtonian heating," World Academy of Science, Engineering and Technology, vol. 64, pp. 628-633, 2012.

[17] R. C. Chaudhary and P. Jain, "Unsteady free convection boundary layer flow past an impulsively started vertical surface with Newtonian heating," Romanian Journal of Physics, vol. 51, pp. 911-925, 2006.

[18] P. Mebine and E. M. Adigio, "Unsteady free convection flow with thermal radiation past a vertical porous plate with newtonian heating," Turkish Journal of Physics, vol. 33, no. 2, pp. 109119, 2009.

[19] M. Narahari and A. Ishak, "Radiation effects on free convection flow near a moving vertical plate with newtonian heating," Journal of Applied Sciences, vol. 11, no. 7, pp. 1096-1104, 2011.

[20] M. Narahari and M. Yunus Nayan, "Free convection flow past an impulsively started infinite vertical plate with Newtonian heating in the presence of thermal radiation and mass diffusion,"
Turkish Journal of Engineering and Environmental Sciences, vol. 35, no. 3, pp. 187-198, 2011.

[21] V. Rajesh, "Effects of mass transfer on flow past an impulsively started infinite vertical plate with Newtonian heating and chemical reaction," Journal of Engineering Physics and Thermophysics, vol. 85, no. 1, pp. 221-228, 2012.

[22] P. Chandrakala and P. N. Bhaskar, "Radiation effects on oscillating vertical plate with uniform heat flux and mass diffusion," International Journal of Fluids Engineering, vol. 4, pp. 1-11, 2012.

[23] R. Muthucumaraswamy, P. Chandrakala, and S. A. Raj, "Radiative heat and mass transfer effects on moving isothermal vertical plate in the presence of chemical reaction," International Journal of Applied Mechanics and Engineering, vol. 11, no. 3, pp. 639-646, 2006.

[24] R. Muthucumaraswamy and A. Vijayalakshmi, "Effects of heat and mass transfer on flow past an oscillating vertical plate with variable temperature," International Journal of Applied Mathematics and Mechanics, vol. 4, no. 1, pp. 59-65, 2008.

[25] V. R. Prasad, N. B. Reddy, and R. Muthucumaraswamy, "Radiation and mass transfer effects on two-dimensional flow past an impulsively started infinite vertical plate," International Journal of Thermal Sciences, vol. 46, no. 12, pp. 1251-1258, 2007.

[26] R. Siegel and J. R. Howell, Thermal Radiation Heat Transfer, Taylor \& Francis, New York, NY, USA, 4th edition, 2002.

[27] E. Magyari and A. Pantokratoras, "Note on the effect of thermal radiation in the linearized Rosseland approximation on the heat transfer characteristics of various boundary layer flows," International Communications in Heat and Mass Transfer, vol. 38, no. 5, pp. 554-556, 2011.

[28] R. A. Mohamed, S. M. Abo-Dahab, and T. A. Nofal, “Thermal radiation and MHD effects on free convective flow of a polar fluid through a porous medium in the presence of internal heat generation and chemical reaction," Mathematical Problems in Engineering, vol. 2010, Article ID 804719, 2010. 


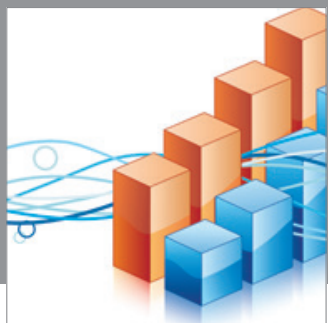

Advances in

Operations Research

mansans

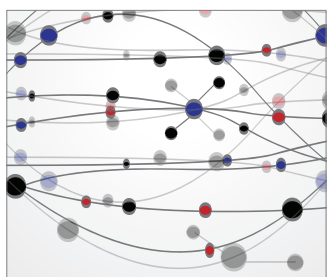

The Scientific World Journal
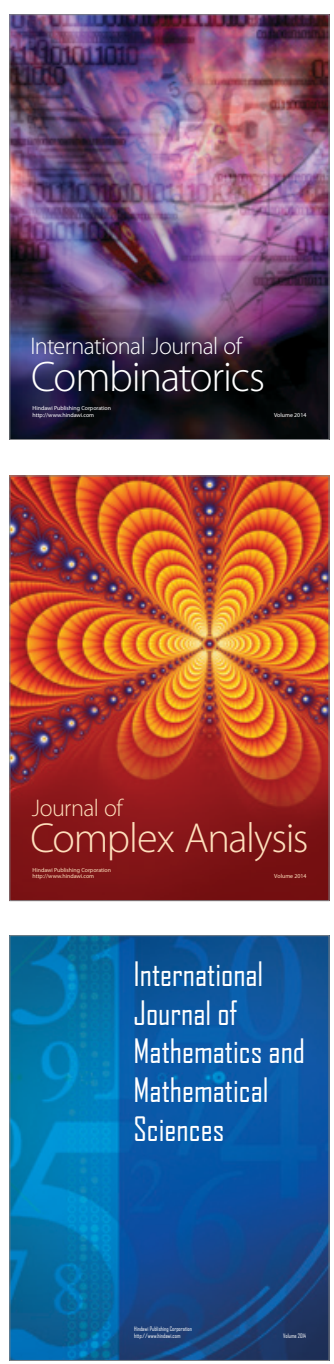
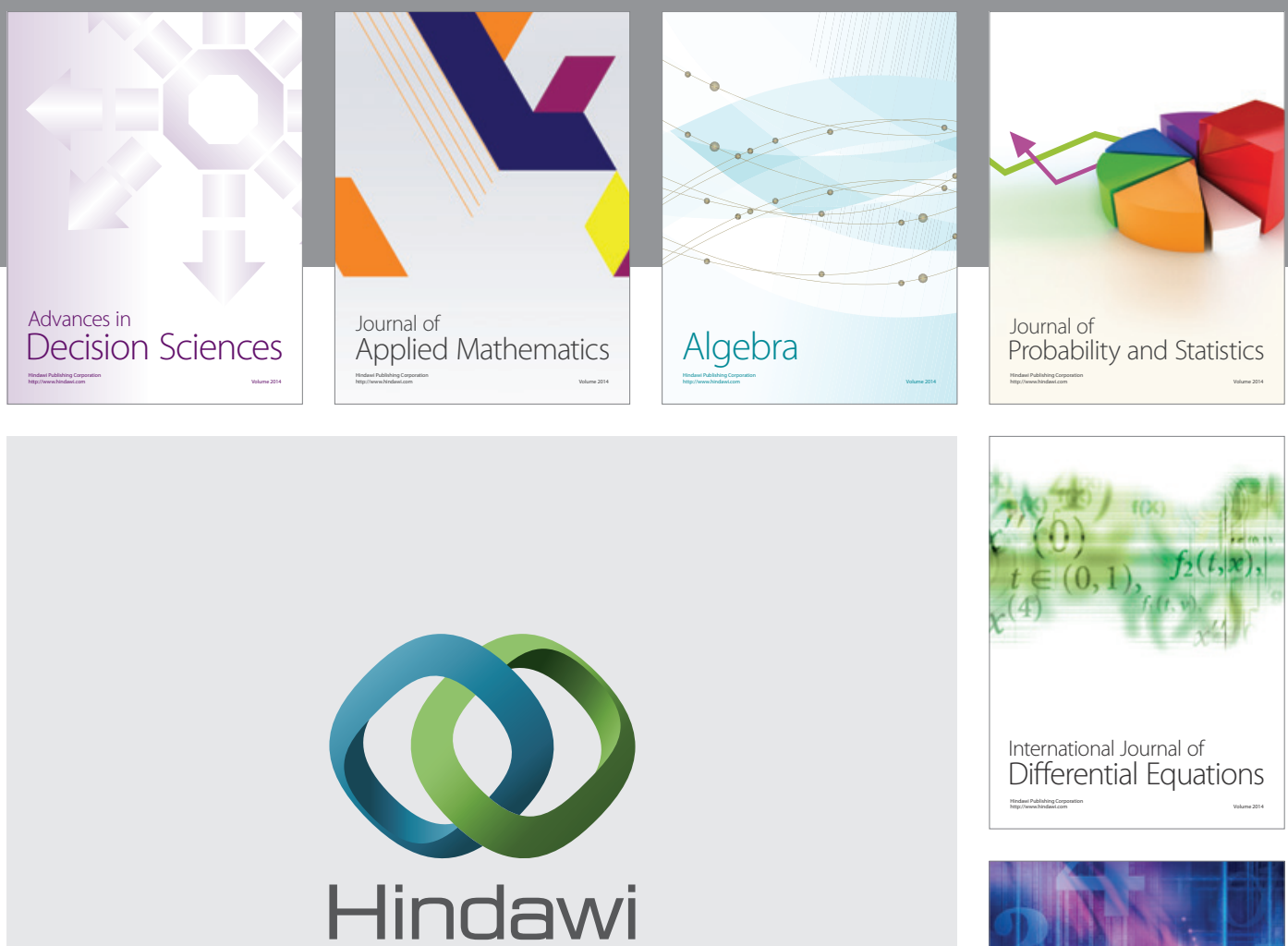

Submit your manuscripts at http://www.hindawi.com
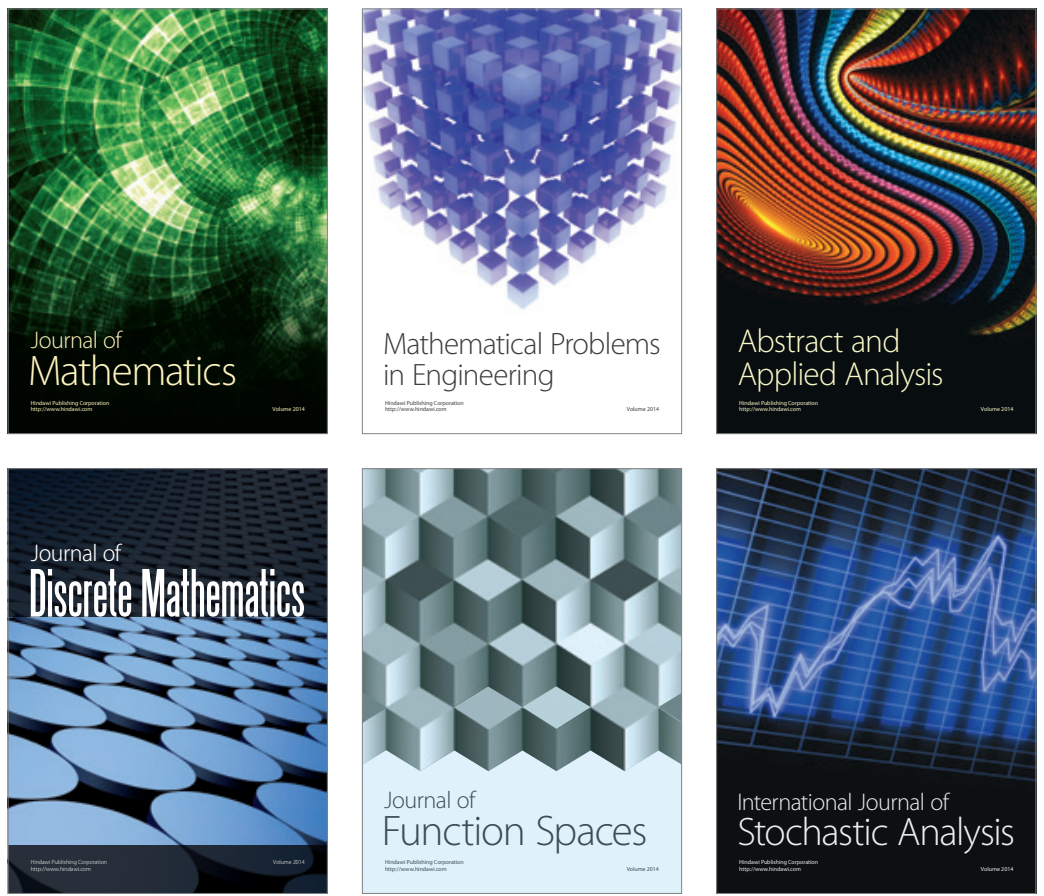

Journal of

Function Spaces

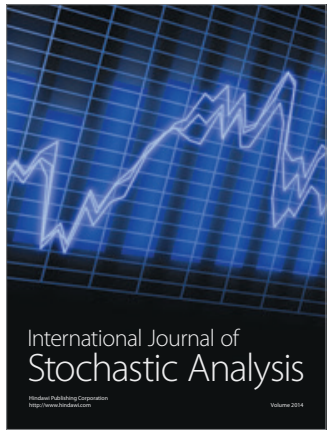

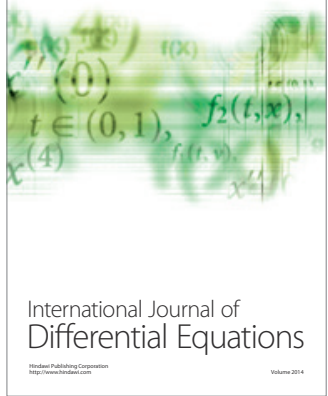
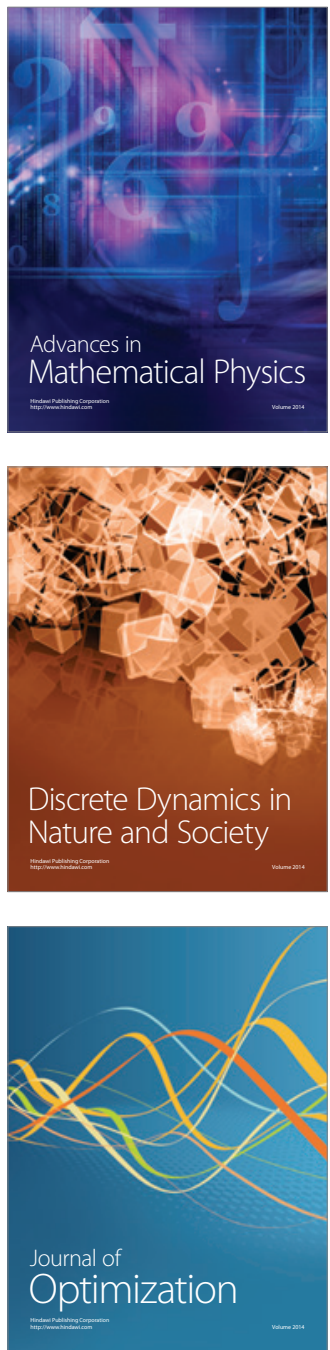